\title{
THE $(1,2)$ CORRESPONDENCE ASSOCIATED WITH THE CUBIC SPACE INVOLUTION OF ORDER TWO*广
}

BY

\section{F. R. SHARPE AND VIRGIL SNYDER}

1. In two previous papers $\ddagger$ we have developed a process of obtaining $(1,2)$ correspondences between two spaces from which rational space involutions can be derived. In the present paper we consider the problem of finding the $(1,2)$ correspondence associated with a given rational space involution and particularly with the non-monoidal cubic involution of order two. The results obtained are of special interest on account of the configuration of the fundamental elements.

2. Consider an involution in a space $x$ such that the pairs of conjugate points are $(y),\left(y^{\prime}\right)$. We are concerned with the birational transformation which interchanges the points of each pair. The lines joining conjugate points $(y),\left(y^{\prime}\right)$ constitute either a congruence or a complex. In the former case each line contains an infinite number of pairs; in the latter, a finite number of pairs lies on each line of the complex. In the case of the cubic involution, the point $\left(y^{\prime}\right)$ is the point of intersection of the polar planes of the point $(y)$ with respect to three quadrics $A(x)=0, B(x)=0$, $C(x)=0$. The quadric of the net that contains the line $(y)\left(y^{\prime}\right)$ has the equation

$$
\left|\begin{array}{lll}
A(x) & B(x) & C(x) \\
A(y) & B(y) & C(y) \\
A\left(y^{\prime}\right) & B\left(y^{\prime}\right) & C\left(y^{\prime}\right)
\end{array}\right|=0
$$

There is one point $(X)$ on the line $(y)\left(y^{\prime}\right)$ such that the tangent plane at this point to the quadric (1) shall pass through $P_{8}$, a selected one of the eight basis points of the net of quadrics. Conversely, given a point $(X)$, there is

* The investigation upon which this article is based was supported in part by a grant from the Heckscher Foundation for the Advancement of Research, established by August Heckscher at Cornell University. We also wish to express our indebtedness to Professor Gino Fano, of Turin University, for helpfull suggestions.

t Presented to the Society, September 7, 1922.

$\ddagger$ Certain types of involutorial space transformations, these Transactions, vol.20(1919), pp. 185-202 and vol. 21 (1920), pp. 52-78. 
one quadric of the net (1) that contains the line $(X) P_{8}$, and on this quadric passes through $(X)$ a unique second line on the quadric. On this line is just one pair of conjugate points in the involution I, namely, the points in which the line is met by its conjugate cubic curve in $I$. Hence the lines $(y)\left(y^{\prime}\right)$ constitute a complex, and this complex is rational; moreover, since there is a $(1,2)$ correspondence between the points $(X)$ and the points $(y)$, the cubic involution $I$ is also rational.

By choosing four of the eight basis points as the vertices of the tetrahedron of reference we may take for three quadrics of the net

$$
\begin{aligned}
& A(x)=x_{4} x_{1}+a_{1} x_{2} x_{3}+a_{2} x_{3} x_{1}+a_{3} x_{1} x_{2}=0 \\
& B(x)=x_{4} x_{2}+b_{1} x_{2} x_{3}+b_{2} x_{3} x_{1}+b_{3} x_{1} x_{2}=0 \\
& C(x)=x_{4} x_{3}+c_{1} x_{2} x_{3}+c_{2} x_{3} x_{1}+c_{3} x_{1} x_{2}=0
\end{aligned}
$$

Let $P_{8}=(0,0,0,1)$. The tangent plane to $(1)$ at $P_{8}$ contains. $(X)$. If we write

$$
X_{i}=k^{\prime} y_{i}+k y_{i}^{\prime}
$$

we find, by putting $(X)$ for $(x)$ in the equation of the tangent plane, that

$$
k=\left|\begin{array}{ccc}
y_{1} & y_{\mathrm{a}} & y_{\mathrm{s}} \\
A(y) & B(y) & C(y) \\
A\left(y^{\prime}\right) & B\left(y^{\prime}\right) & C\left(y^{\prime}\right)
\end{array}\right|
$$

and

$$
-k^{\prime}=\left|\begin{array}{ccc}
y_{1}^{\prime} & y_{\mathrm{a}}^{\prime} & y_{\mathrm{s}}^{\prime} \\
A(y) & B(y) & C(y) \\
A\left(y^{\prime}\right) & B\left(y^{\prime}\right) & C\left(y^{\prime}\right)
\end{array}\right|
$$

The three polar relations $A\left(y, y^{\prime}\right)=0, B\left(y, y^{\prime}\right)=0, C\left(y, y^{\prime}\right)=0$ determine the values of $y_{i}^{\prime}$ as cubic functions of $(y)$. The surfaces $y_{i}^{\prime}=0$ have in common a sextic curve $\gamma_{6}$, locus of the vertices of the quadric cones contained in the net of quadrics. It follows that $k=0$ contains $\gamma_{6}$ triply, and $k^{\prime}$ contains $\gamma_{6}$ doubly, and the surfaces $X_{i}=0$, of order 12 , contain $\gamma_{6}$ triply. Since $k=0$ is of order 11 , and $k^{\prime}=0$ is of order 9 , it follows further that apart from $\gamma_{6}$, the surfaces (3) have in common a curve of order 63. Hence two surfaces of the system (3) meet in a variable curve of order 27.

For our purpose, it will be convenient to use the following notation. Let a general quadric of the net be expressed in the form

$$
\lambda_{1} A(x)+\lambda_{2} B(x)+\lambda_{3} C(x)=0 .
$$


It will contain the points $(y),\left(y^{\prime}\right)$ when

$$
\begin{aligned}
& \lambda_{1} A(y)+\lambda_{2} B(y)+\lambda_{3} C(y)=0, \\
& \lambda_{1} A\left(y^{\prime}\right)+\lambda_{2} B\left(y^{\prime}\right)+\lambda_{3} C\left(y^{\prime}\right)=0 .
\end{aligned}
$$

If we now write $p_{i k}=y_{i} y_{k}^{\prime}-y_{k} y_{i}^{\prime}$ we may express equations (3) in the form

$$
\begin{aligned}
& X_{1}=p_{12} \lambda_{2}+p_{13} \lambda_{3}, \\
& X_{2}=p_{21} \lambda_{1}+p_{23} \lambda_{3}, \\
& X_{3}=p_{31} \lambda_{1}+p_{32} \lambda_{2}, \\
& X_{4}=p_{41} \lambda_{1}+p_{42} \lambda_{2}+p_{43} \lambda_{3} .
\end{aligned}
$$

The surfaces $p_{i k}=0$ are of order four, and pass through $\gamma_{6}$ simply. From (5) we see that the surfaces $\lambda_{i}=0$ are of order 8 and pass through $\gamma_{6}$ doubly.

3. Fundamental elements. Consider a point $\left(1, y_{2}, y_{3}, y_{4}\right)$ near the basis point $P_{1}=(1,0,0,0)$. From the polar relations $A\left(y, y^{\prime}\right)=0$, $B\left(y, y^{\prime}\right)=0, C\left(y, y^{\prime}\right)=0$ it follows that $\left(y^{\prime}\right)=\left(1,-y_{2},-y_{3},-y_{4}\right)$ to the first order of small numbers; hence

$$
X_{2}=2 y_{2}, X_{3}=2 y_{3}, X_{4}=2 y_{4} .
$$

From the relation $\lambda_{1} A(y)+\lambda_{2} B(y)+\lambda_{3} C(y)=0$ we have

$$
\lambda_{1}\left(y_{4}+a_{2} y_{3}+a_{3} y_{2}\right)+\lambda_{2}\left(b_{z} y_{3}+b_{3} y_{z}\right)+\lambda_{3}\left(c_{z} y_{3}+c_{3} y_{z}\right)=0 ;
$$

hence, from the preceding relations,

$$
\lambda_{1}\left(X_{4}+a_{2} X_{3}+a_{3} X_{2}\right)+\lambda_{2}\left(b_{2} X_{3}+b_{3} X_{2}\right)+\lambda_{3}\left(c_{2} X_{3}+c_{3} X_{2}\right)=0 .
$$

Moreover, since $\lambda_{1} A(x)+\lambda_{2} B(x)+\lambda_{3} C(x)=0$ contains the line

we also have

$$
\frac{x_{1}}{X_{1}}=\frac{x_{2}}{X_{2}}=\frac{x_{3}}{X_{3}}
$$

$$
\begin{gathered}
\lambda_{1} X_{1}+\lambda_{2} X_{2}+\lambda_{3} X_{3}=0 \\
\lambda_{1}\left(a_{1} X_{2} X_{3}+a_{2} X_{3} X_{1}+a_{3} X_{1} X_{2}\right)+\lambda_{2}\left(b_{1} X_{2} X_{3}+b_{2} X_{3} X_{1}+b_{3} X_{1} X_{2}\right) \\
+\lambda_{3}\left(c_{1} X_{2} X_{3}+c_{2} X_{3} X_{1}+c_{3} X_{1} X_{2}\right)=0
\end{gathered}
$$

Upon eliminating $\lambda_{1}, \lambda_{2}, \lambda_{3}$ from these three equations we obtain as image of the basis points $P_{1}=(1,0,0,0)$ a quartic surface. 
Now consider the quartic surface $p_{23}=0$, that has the equation

$$
\left|\begin{array}{cccc}
x_{4}+a_{2} x_{3}+a_{3} x_{2} & a_{1} x_{3}+a_{3} x_{1} & a_{1} x_{2}+a_{2} x_{1} & x_{1} \\
b_{2} x_{3}+b_{3} x_{2} & x_{4}+b_{1} x_{3}+b_{3} x_{1} & b_{1} x_{2}+b_{2} x_{1} & x_{2} \\
c_{2} x_{3}+c_{3} x_{2} & c_{1} x_{3}+c_{3} x_{1} & x_{4}+c_{1} x_{2}+c_{2} x_{1} & x_{3} \\
0 & -x_{3} & x_{2} & 0
\end{array}\right|=0 .
$$

By multiplying the columns by $x_{1}, x_{2}, x_{3}, x_{1}$ respectively and adding to the second we obtain

$$
\left|\begin{array}{cccc}
x_{4}+a_{2} x_{3}+a_{3} x_{2} & A(x) & a_{1} x_{2}+a_{2} x_{1} & x_{1} \\
b_{2} x_{3}+b_{3} x_{2} & B(x) & b_{1} x_{2}+b_{2} x_{1} & x_{2} \\
c_{2} x_{3}+c_{3} x_{2} & C(x) & x_{4}+c_{1} x_{2}+c_{2} x_{1} & x_{3} \\
0 & 0 & x_{2} & 0
\end{array}\right|=0 .
$$

After removing the extraneous factor $x_{2}$ the result is exactly of the same form in $(x)$ as the image in $(X)$ of the point $P_{1}$ in $(y)$.

The surface $p_{23}=0$ has $P_{8} P_{1}$ for double line; the lines joining each of these points to the other six basis points lie on the surface.

The correspondence between $(y)$ and $(X)$ is clearly symmetrical in the seven points $P_{i}$, hence the image of each of them is a quartic surface. By introducing the notation (81) for the surface having the line $P_{8} P_{1}$ for double line, we can readily write down the complete configuration of the lines on each of the seven surfaces.

4. The twenty-one basis lines in $(y)$. The lines joining the seven basis points $P_{i}, i \neq 8$, to each other belong to every surface of the web of $s_{12}$. Consider the point $\left(y_{1}, y_{2}, 0,0\right)$ on the line $y_{8}=0, y_{4}=0$. The conjugate point $\left(y^{\prime}\right)$ in $I$ is $\left(y_{2},-y_{1}, 0,0\right)$, hence the line is invariant in $I$ and no points are fundamental except the two basis points which it contains. Since the line contains an infinite number of pairs of conjugates, it also belongs to a whole pencil of quadrics of the web, given by the equation

$$
\lambda_{1} a_{3}+\lambda_{9} b_{3}+\lambda_{3} c_{3}=0
$$

and the associated value of $(X)$ is $\left(\lambda_{2},-\lambda_{1}, 0,0\right)$.

Hence the image of a point $(y)$ on any line in $(y)$ is the whole line in $(X)$; conversely, the image in $(y)$ of any point $(X)$ on one of the lines in $(X)$ is the whole line in $(y)$.

5. The fundamental lines $l(X)$. We shall denote by $l$ the lines joining $P_{8}$ to the other seven basis points of the net of quadrics. Consider the point $(X)$ near $\left(0,0, X_{8}, X_{4}\right)$. We have $\lambda_{3}=0$ and $\lambda_{1} A(x)=\lambda_{8} B(x)=0$ for 
the pencil of quadrics. The tangent plane to the quadric at $(X)$ is [ $\left(X_{4}+a_{2} X_{3}\right)$ $\left.+b_{8} X_{3}\right] x_{1}+\left[a_{1} X_{3}+\left(X_{4}+b_{1} X_{3}\right)\right] x_{8}=0$. If we eliminate $\lambda_{1}: \lambda_{8}$ we have a cubic cone on which lie the points $(y)\left(y^{\prime}\right)$; moreover

$$
\frac{y_{1}}{y_{2}}=\frac{y_{1}^{\prime}}{y_{2}^{\prime}}
$$

hence the image of the line $X_{1}=0, X_{2}=0$ is the quartic $y_{1} y_{2}^{\prime}=y_{1}^{\prime} y_{2}$, that is, $p_{18}=0$. The image in $(y)$ of a point on this line $l(X)$ is a curve of order eight in which the cubic cone and the quadric meet apart from the line $y_{1}=0$, $y_{8}=0$. Similarly for each of the seven lines joining $P_{8}$ to each of the other seven basis points.

The image in $(y)$ of any line joining $P_{8}$ to one of the other basis points $P_{i}$, considered as a line of $(X)$ is a quartic surface, having the line defined by the same equations in $(y)$ for double line, and containing the six lines through each of the basis points on the line which join it to the other basis points. Moreover, all of these seven quartic surfaces pass through the curve $\gamma_{6}$.

Any two of these quartic surfaces meet in $\gamma_{6}$, in their respective double lines, counting for four, in the other five lines $l(y)$, and in the line $m(y)$ joining the basis points on their double lines.

6. The fundamental curve $\gamma_{6}$ in $(X)$. Any point $(X)$ of $\gamma_{6}$ is the vertex of a quadric cone belonging to the net; every generator of this cone contains a pair of conjugate points $(y)\left(y^{\prime}\right)$, hence the image of $(X)$ is a curve lying on its quadric cone. There are two positions of $(y)$ the conjugates of which in I coincide with any given point on $\gamma_{6}$, because the conjugate of a point $(X)$ on $\gamma_{6}$ is every point of a straight line, which is a bisecant of $\gamma_{6}$ and meets the cone in two points. Hence the image curve of $(X)$ has a double point at $(X)$. Since any plane passing through $(X)$ meets the cone in two generators, each of which contains two image points, it follows that the curve is a sextic, having a double point at $(X)$. The locus of this sextic curve, as $(X)$ describes $\gamma_{6}$, is a surface, the order of which is determined as follows: the jacobian of the web of $s_{12}$ is of order 44, and it consists of the seven quartic surfaces $p_{i k}=0$, and of the image of $\gamma_{6}$, which is therefore of order 16. This surface $\Gamma_{16}$ has all the 28 lines joining basis points for double lines, contains $\gamma_{6}$ four-fold; the point $P_{8}$ is eight-fold, the other seven basis points being six-fold.

7. Images of the plane $X_{i}=0, i=1,2,3$. The image of the plane $X_{1}=0$ consists of the two quartics (82), (83), and of a residual quartic. Since (82) has the line $P_{8} P_{8}$ double, and the lines joining either $P_{8}$ or $P_{8}$ to the other six basis points simple, and similarly (83) contains the line $P_{8} P_{8}$ doubly, and the lines joining either $P_{8}$ or $P_{8}$ to each of the other basis points simply, and since the configuration of multiple lines is symmetric in all the lines on $s_{18}$, it follows that the residual quartic surface, image of the plane $X_{1}=0$, apart 
from the fundamental lines in it, contains the lines joining $P_{8}$ to the other five basis points, the four lines joining the remaining vertex of the tetrahedron of reference to the four remaining basis points, and the six edges of the tetrahedron formed by the basis points not at the vertices of the tetrahedron of reference. Similarly for $X_{2}=0, X_{3}=0$.

We shall now prove analytically that the image of any plane of the bundle $k_{1} X_{1}+k_{8} X_{8}+k_{3} X_{3}=0$ has the seven lines $l(y)$ triple and the twenty-one lines $m(y)$ simple. From the polar relations $A\left(y, y^{\prime}\right)=0, B\left(y, y^{\prime}\right)=0$, we have

$y_{4} y_{1}^{\prime}+a_{1} y_{2}^{\prime} y_{3}+a_{2} y_{3} y_{1}^{\prime}+a_{3} y_{1} y_{2}^{\prime}=-\left(y_{4}^{\prime} y_{2}+a_{1} y_{8} y_{8}^{\prime}+a_{2} y_{8}^{\prime} y_{1}+a_{3} y_{2} y_{1}^{\prime}\right)$, $y_{4}^{\prime} y_{2}+b_{1} y_{2} y_{3}^{\prime}+b_{8} y_{3}^{\prime} y_{1}+b_{3} y_{1}^{\prime} y_{2}=-\left(y_{4} y_{2}^{\prime}+b_{1} y_{2}^{\prime} y_{3}+b_{8} y_{3} y_{1}^{\prime}+b_{3} y_{2}^{\prime} y_{1}\right)$.

Multiplying the two equations together, member by member, then transposing the second member to the right, and subtracting the result from the value of $\lambda_{3}=A(y) B\left(y^{\prime}\right)-A\left(y^{\prime}\right) B(y)$, we obtain

$$
\begin{aligned}
\lambda_{3}=p_{18} & {\left[2 y_{4} y_{4}^{\prime}+\left(a_{2}+b_{1}\right)\left(y_{4} y_{3}^{\prime}+y_{3} y_{4}^{\prime}\right)+b_{3}\left(y_{4} y_{1}^{\prime}+y_{4}^{\prime} y_{1}\right)\right.} \\
& \left.+2\left(a_{1} b_{2}\right) y_{3} y_{3}^{\prime}+\left(a_{2} b_{3}\right)\left(y_{3} y_{1}^{\prime}+y_{3}^{\prime} y_{1}\right)\right] .
\end{aligned}
$$

Eliminating the terms $y_{4} y_{1}^{\prime}+y_{4}^{\prime} y_{1}$ by means of $A\left(y, y^{\prime}\right)=0$, we may write

$$
\begin{gathered}
\frac{\lambda_{3}}{p_{12}}=2 y_{4} y_{4}^{\prime}+\left(a_{2}+b_{1}\right)\left(y_{4} y_{8}^{\prime}+y_{3} y_{4}^{\prime}\right)+2\left(a_{1} b_{8}\right) y_{3} y_{8}^{\prime}-a_{3} b_{2}\left(y_{3} y_{1}^{\prime}+y_{3}^{\prime} y_{1}\right) \\
-b_{3} a_{1}\left(y_{3} y_{2}^{\prime}+y_{2} y_{3}^{\prime}\right)-a_{3} b_{3}\left(y_{1} y_{2}^{\prime}+y_{2} y_{1}^{\prime}\right)=\mu_{3} .
\end{gathered}
$$

Similar expressions can be found for $\mu_{1}=\lambda_{1} / p_{23}$ and $\mu_{2}=\lambda_{2} / p_{31}$, hence the correspondence (6) may now be written in the form

$$
\begin{aligned}
X_{1} & =p_{12} p_{31}\left(\mu_{2}-\mu_{3}\right), \\
X_{2} & =p_{23} p_{18}\left(\mu_{3}-\mu_{1}\right), \\
X_{3} & =p_{31} p_{28}\left(\mu_{1}-\mu_{2}\right), \\
X_{4} & =p_{41} p_{28} \mu_{1}+p_{48} p_{31} \mu_{2}+p_{43} p_{12} \mu_{8} \\
& =p_{41} p_{28}\left(\mu_{1}-\mu_{8}\right)+p_{48} p_{31}\left(\mu_{2}-\mu_{3}\right) .
\end{aligned}
$$

It is now readily verified that the seven central lines $l(y)$ are triple on the surfaces $X_{1}=0, X_{2}=0, X_{8}=0$, and double on $X_{4}=0$, while the twentyone lines $m(y)$ are simple on all the surfaces of the web. The basis curve of order 63 is therefore made up of the seven central lines $l(y)$, each counted six times, and the 21 lines $m(y)$.

The tangent planes to the surfaces of the web at each point of the seven lines are fixed. Moreover, it can be shown that for the image of the plane $X_{4}=0$ the tangent cone at $P_{8}$ is of order six, while the tangent cone of the image of any plane of the bundle through $(0,0,0,1)$ at $P_{8}$ is of order eight. 
Hence the curve of order 27, image of a general line of $(X)$, has six variable branches through $P_{8}$ lying on the tangent cone of the image of $X_{4}=0$. The image of the point $P_{8}(y)$ is therefore a surface of order 6 in $(X)$.

Moreover, the image in $(X)$ of a plane in $(y)$ is a surface of order 27, having the seven central lines $l(X)$ eight-fold, since the image of a point of one of these lines is a curve of order 8 in $(y)$. The curve $\gamma_{6}$ is 6 -fold on all the surfaces of the system, for a similar reason.

This surface $s_{27}^{\prime}$ is the image of a plane in $(y)$ and also of the conjugate cubic surface of the plane in the involution $I$; hence it follows that the image of the curve $\gamma_{6}(y)$ is a surface $\Gamma_{54}^{\prime}$ of order 54 in $(X)$, having the seven central lines $l(X) 16$-fold and the curve $\gamma_{6}(X) 12$-fold. The complete image in $(y)$ of this surface $\Gamma_{54}^{\prime}$ is the curve $\gamma_{6}(y)$ and its conjugate ruled surface of order 8 in $\mathrm{I}$. Hence the image in $(y)$ of a straight line in $(X)$ is a curve of order 27, having a six-fold point at $P_{8}(y)$, a four-fold point at each of the other basis points and meeting $\gamma_{6}(y)$ in 54 points. The curve meets a general surface of the web $s_{19}$ in $12 \cdot 27-4 \cdot 4 \cdot 7-6 \cdot 8-54 \cdot 3=2$ variable intersections. The point $P_{8}$ counts for 48 intersections on account of the fixed sextic cone at $P_{8}$.

This is verified by replacing the curve of order 27 by the image of a line through $P_{8}(X)$. Each of the seven central lines $l(y)$ now counts for 9 in the intersection of the image surfaces; hence the image of any such line is a curve of order 6 meeting $\gamma_{6}$ in 12 points and passing simply through the eight basis points $P_{i}$. The sextic curve meets the image of a general plane of $(X)$ in $6 \cdot 12-32 \cdot 3-7 \cdot 4-6=2$ variable points. Since a line through $P_{8}(X)$ has a sextic curve for image, the image of $P_{8}$ itself consists of the seven central lines $l(y)$, each counted three times.

7. Using the notation previously employed, we may write

$$
\begin{aligned}
& s_{1}^{\prime} \cos s_{18}: \gamma_{8}^{8}+7 l+21 m, \\
& c_{1}^{\prime} \cos c_{87}: P_{8}^{8}+7 P_{i}^{4},\left[c_{27}, \gamma_{6}\right]=54, \\
& s_{1} \cos s_{27}^{\prime}: \gamma_{8}^{18}+7 l^{\prime 8}, \\
& c_{1} \cos c_{12}^{\prime}:\left[c_{12}^{\prime}, l^{\prime}\right]=4, \\
& \gamma_{6} \cos \Gamma_{54}^{\prime}: \gamma_{6}^{\prime 18}+7 l^{\prime 16}, \\
& \gamma_{6}^{\prime} \cos \Gamma_{16}: \gamma_{8}^{4}+P_{8}^{8}+7 P_{i}^{8}+7 l^{2}+21 m^{2}, \\
& l \cos A_{4}^{\prime}: l^{2}+6 l^{\prime}+6 m^{\prime}+\gamma_{6}^{\prime}, \\
& P_{i}^{\prime} \cos (8 i)_{4}: l_{i}^{8}+6 l+6 m+\gamma_{6}, \\
& P_{8} \cos s_{6}^{\prime}, \\
& X_{i}=0 \cos s_{18}: \gamma_{6}^{8}+7 l^{3}+21 m, \quad i=1,2,3, \\
& X_{4}=0 \cos s_{18}: \gamma_{8}^{8}+7 l^{2}+21 m, \\
& P_{8}^{\prime} \cos 7 l^{3} .
\end{aligned}
$$


8. The cubic inversion. If the quadrics $A(x)=0, B(x)=0, C(x)=0$ have the special forms $x_{1} x_{4}+x_{2} x_{3}=0, x_{2} x_{4}+x_{3} x_{4}=0, x_{3} x_{4}+x_{1} x_{2}=0$, then the four basis points, apart from the vertices of the tetrahedron of reference, are $(1,1,1,-1),(1,1,-1,1),(1,-1,1,1),(-1,1,1,1)$ respectively. This case can be discussed more easily if we change the coordinate system to the self-polar tetrahedron of the three quadrics. We may then take three quadrics of the form $x_{1}^{2}-x_{4}^{2}=0, x_{8}^{2}-x_{4}^{2}=0, x_{8}^{2}-x_{4}^{2}=0$, so that the net of quadrics has the form $\sum \lambda_{i} x_{i}^{2}=0$, where $\sum \lambda_{i}=0$.

The involution is now defined by the equations

$$
y_{1} y_{1}^{\prime}=y_{2} y_{2}^{\prime}=y_{3} y_{8}^{\prime}=y_{4} y_{4}^{\prime}
$$

The equation of the quadric through $(y)$ and $\left(y^{\prime}\right)$ is given by $\sum \lambda_{i} y_{i}^{2}=0$, $\sum \lambda_{i} y_{i}^{\prime 2}=0$ and is therefore

$$
\left|\begin{array}{cccc}
x_{1}^{2} & x_{2}^{2} & x_{3}^{2} & x_{4}^{2} \\
y_{1}^{2} & y_{3}^{2} & y_{3}^{2} & y_{t}^{2} \\
y_{1}^{\prime 2} & y_{2}^{\prime 2} & y_{3}^{\prime 2} & y_{4}^{\prime 2} \\
1 & 1 & 1 & 1
\end{array}\right|=0 .
$$

The basis points are now $(1, \pm 1, \pm 1, \pm 1)$. We shall take for $P_{8}$ the basis point $(1,1,1,1)$. The tangent plane at $\left(k y+k^{\prime} y^{\prime}\right)$ passes through the point $P_{8}$ if $\sum \lambda_{i}\left(k y_{i}+k^{\prime} y_{i}^{\prime}\right)=0$; hence

so that

$$
\frac{k}{k^{\prime}}=-\frac{\sum \lambda_{i} y_{i}^{\prime}}{\sum \lambda_{i} y_{i}}
$$

$$
X_{1}=\lambda_{2} p_{12}+\lambda_{3} p_{13}+\lambda_{4} p_{14},
$$

and similarly for $X_{2}, X_{3}, X_{4}$.

We find

$$
\lambda_{1}=\left(y_{\mathrm{a}}^{2}-y_{\mathrm{8}}^{2}\right)\left(y_{\mathrm{z}}^{2}-y_{4}^{2}\right)\left(y_{4}^{2}-y_{\mathrm{a}}^{2}\right) y_{1}^{2},
$$

and similarly for $\lambda_{2}, \lambda_{3}, \lambda_{4}$, so that

$$
k^{\prime}=\prod\left(y_{1}-y_{2}\right) \sum y_{1} y_{2} y_{3}, \quad k=-\prod\left(y_{1}-y_{2}\right) \sum y_{1} \cdot y_{1} y_{2} y_{3} y_{4} .
$$

Hence

$$
X_{1}=\left(y_{1}+y_{2}\right)\left(y_{1}+y_{3}\right)\left(y_{1}+y_{4}\right) y_{2} y_{3} y_{4},
$$

and similarly for $X_{2}, X_{3}, X_{4}$.

The correspondence is therefore of order 6 , the images in $(y)$ of the planes of $(X)$ being surfaces of Enriques* having the six edges of the tetrahedron of reference for double curve. These edges constitute the $\gamma_{6}$ of the general case.

* See Picard et Sinart, Théorie des Fonctions algébriques de deux Variables indépendents, vol. 2, p. 148. 
The surfaces also contain the lines $y_{1}+y_{2}=0, y_{3}+y_{4}=0$, and two similar lines. Two surfaces of the web meet in a variable curve of order 9 , image of a line in $(X)$.

9. Fundamental elements in $(y)$. The image in $(X)$ of the point $(1,1,1,1)$ in $(y)$ is clearly the point $(1,1,1,1)$. To obtain the image of another basis point, for example $(-1,1,1,1)$, consider a neighboring point $(-1,1+\beta, 1+\gamma, 1+\delta)$. We at once find $X_{1}=0$. Similarly for the other three points of this type. Next, consider the point $(1,1+\beta,-1+\gamma$, $-1+\delta)$ near the basis point $(1,1,-1,-1)$. Here we find $X_{1}=2 \gamma \delta$, $X_{2}=2(\beta+\gamma)(\beta+\delta), X_{3}=-2 \gamma(\beta+\gamma), X_{4}=-2 \delta(\beta+\delta)$. Hence the image surface is $X_{3} X_{4}-X_{1} X_{2}=0$. Similarly for the other two points of this type.

The composite curve $\gamma_{6}$. To obtain the image in $(X)$ of a line $y_{1}=0$, $y_{2}=0$, consider a point $(y)$ near the line, and retain only the lowest powers of $y_{1}$ and $y_{2}$. Thus

$$
\begin{array}{ll}
X_{1}=y_{2}\left(y_{1}+y_{2}\right) y_{3} y_{4}, & X_{2}=y_{1}\left(y_{1}+y_{2}\right) y_{3} y_{4}, \\
X_{3}=y_{1} y_{2} y_{3}\left(y_{3}+y_{4}\right), & X_{4}=y_{1} y_{2} y_{4}\left(y_{3}+y_{4}\right),
\end{array}
$$

hence by eliminating $y_{1}$ and $y_{2}$ we have the image of the point. The point $\left(0,0, y_{3}, y_{4}\right)$ has for image the curve $\left(y_{3}+y_{4}\right) X_{1} X_{2}-y_{4} X_{3}\left(X_{1}+X_{2}\right)=0$, $y_{4} X_{3}=y_{3} X_{4}$. The image of the whole line is obtained by eliminating $y_{3}, y_{4}$ from these equations. The image of the line $y_{1}=0, y_{2}=0$ and also of the line $y_{3}=0, y_{4}=0$ is therefore the cubic surface $X_{1} X_{2}\left(X_{3}+X_{4}\right)=$ $X_{3} X_{4}\left(X_{1}+X_{2}\right)$. Similarly for the other four edges of the tetrahedron of reference.

The line $y_{1}+y_{2}=0, y_{3}+y_{4}=0$. Proceeding as before, take a point $\left(y_{1},-y_{1}+p, y_{3},-y_{3}+q\right)$ near the line and retain only the lowest powers of the small numbers $p, q$. After discarding a common factor we find

$$
X_{1}=p y_{3}, X_{2}=-p y_{3}, X_{3}=-q y_{1}, X_{4}=q y_{1},
$$

so that $X_{1}+X_{2}=0, X_{3}+X_{4}=0$. The image of any point on the first line is therefore the whole of the second line. Similarly for the other two basis lines in $(y)$ having a similar form of equations.

The surfaces $s_{6}(y)$ of the web pass through the edges of the tetrahedron of reference doubly, contain the points $(1,1,-1,-1)$, etc. doubly, and the points, $(1,1,1,-1)$, etc. simply. Hence the complete image in $(X)$ of any surface of the web consists of a plane $s_{1}^{\prime}(X)$ counted twice, of six cubic surfaces, each taken twice, of three quadrics; each taken twice, and of the four planes $X_{i}=0$, each taken simply.

10. Fundamental elements in $(X)$. Vertices of the composite curve $\gamma_{6}$. From the expressions for $X_{i}$ in equations (7) in terms of $(y)$ it is clear that 
the point $(0,0,0,1)$ in $(y)$ has for image in $(X)$ the point having the same coördinates. Conversely, therefore, the point $(0,0,0,1)$ in $(X)$ has one image at $(0,0,0,1)$ in $(y)$; the other is the entire plane $y_{4}=0$, since any plane of $(X)$ through this point has for image a sextic surface having the fixed component $y_{4}=0$. The residual quintic surface meets $y_{4}=0$ in a composite quintic consisting of three lines of $\gamma_{6}$ and a variable conic. Hence the point $(0,0,0,1)$ is 5 -fold on $s_{9}^{\prime}$.

11. Lines of the composite curve $\gamma_{6}$. The image of any plane of the pencil through the line $X_{1}=0, X_{2}=0$ has the plane $y_{1}+y_{2}=0$ for fixed component in addition to the planes, images of the vertices on the line. Hence the plane is the image of the line. The sextic surface, image of a general plane in $(X)$, meets the plane $y_{1}+y_{2}=0$ in a cubic curve apart from fixed fundamental elements, hence the curve is the image of a point on the line $X_{1}=0, X_{2}=0$ and the line is triple on every $s_{9}^{\prime}$ of the system.

The point $(1,1,1,1)$. The images of planes through the point $(1,1,1,1)$ have in conımon in addition to fundamental elements common to all the sextic surfaces of the web, the three lines of the type $y_{1}-y_{2}=0, y_{3}-y_{4}=0$; hence the image of the point is these three lines.

The surfaces $s_{9}^{\prime}$ all have the vertices of the composite $\gamma_{6}$ for five-fold points, the edges three-fold. Moreover, they also have the lines of the type $X_{1}-X_{2}=0, X_{3}-X_{4}=0$ double. The image in $(y)$ of an $s_{9}^{\prime}$ of the system is of order 54. It consists of $s_{1}$, its conjugate $s_{3}$ in I, and of the following fundamental elements: the four planes $y_{i}=0$, each counted five times, the six planes $y_{i}+y_{k}=0$, each counted three times, and three quadrics of the type $y_{1} y_{2}-y_{3} y_{4}=0$, each counted twice.

We have seen that any point on the line $y_{1}+y_{2}=0, y_{3}+y_{4}=0$ has for image in $(X)$ the whole line $X_{1}+X_{2}=0, X_{3}+X_{4}=0$. Since any plane in $(y)$ meets the line in one point, its image $s_{9}^{\prime}$ contains the whole line. Hence the system $s_{g}^{\prime}$ has also the three lines of this type for basis elements.

12. Algebraic procedure. The plane containing the points $(y),\left(y^{\prime}\right)$, and the vertex $(1,0,0,0)$ has the equation

$$
p_{34} x_{2}+p_{42} x_{3}+p_{23} x_{4}=0 .
$$

Since $(y)$ and $\left(y^{\prime}\right)$ both satisfy this equation we may write

and

$$
p_{34} y_{2}+p_{42} y_{3}+p_{23} y_{4}=0
$$

hence

$$
\frac{p_{34}}{y_{2}}+\frac{p_{48}}{y_{3}}+\frac{p_{83}}{y_{4}}=0
$$

$$
\frac{y_{4}}{y_{3}}+\frac{y_{3}}{y_{4}}+\frac{p_{23}^{2}+p_{24}^{2}-p_{34}^{2}}{p_{93} p_{34}}=0 \text {. }
$$


Similarly, by considering the plane $(y),\left(y^{\prime}\right),(0,1,0,0)$, we obtain

$$
\frac{y_{4}}{y_{3}}+\frac{y_{3}}{y_{4}}+\frac{p_{18}^{2}+p_{14}^{2}-p_{84}^{2}}{p_{13} p_{14}}=0 \text {. }
$$

If we subtract the latter equation from the former, clear of fractions, and make use of the quadratic identity in $(p)$, we obtain

$$
p_{34} p_{42} p_{23}+p_{41} p_{13} p_{34}+p_{12} p_{24} p_{41}+p_{23} p_{31} p_{12}=0
$$

which is the equation of the cubic complex containing the lines $(y),\left(y^{\prime}\right)$. Conversely, given a line of the cubic complex, we can find a pair of points $(y)$ from the above equations.

Given the point $(X)$, the quadric $\sum \lambda_{i} x_{i}^{2}=0, \sum \lambda_{i}=0$ that contains the line $(X)(1,1,1,1)$ is given by $\sum \lambda_{i} X_{i}=0, \sum \lambda_{i} X_{i}^{q}=0$.

Hence

$$
\lambda_{1}=\left(X_{2}-X_{3}\right)\left(X_{3}-X_{4}\right)\left(X_{4}-X_{2}\right) \text {, etc. }
$$

By considering the intersection of the quadric and its tangent plane at $(X)$ we find

$$
p_{12}=\left(X_{3}-X_{4}\right)\left\{X_{3} X_{4}\left(X_{1}+X_{2}\right)-X_{1} X_{2}\left(X_{3}+X_{4}\right)\right\}
$$

and similarly for the other line coordinates.

Given a plane $A y_{1}+B y_{2}+C y_{3}+D y_{4}=0$, we have $p_{34} y_{2}+p_{12} y_{3}+p_{23} y_{4}=0$ and three similar equations. Hence $y_{1}=B p_{34}+C p_{42}+D p_{23}$ and three similar equations.

By substituting for $\left(y^{\prime}\right)$ in this equation we have the image $s_{9}^{\prime}$ of the given plane in the form

$$
-\left(A^{2}+B^{2}+C^{2}+D^{2}\right) p_{12} p_{18} p_{14}+\sum B C^{2} p_{14}\left(p_{12}^{2}+p_{18}^{2}-p_{14}^{2}\right)=0 .
$$

The factor $\left(X_{3}-X_{4}\right)\left(X_{4}-X_{2}\right)\left(X_{2}-X_{3}\right)$ divides out as can be seen by writing the equation in the form

$$
-(A+B+C+D)^{2} p_{34} p_{12} p_{23}+\sum B C p_{28}\left[\left(p_{34}+p_{48}\right)^{2}-p_{28}^{2}\right]=0,
$$

but $\left(p_{34}+p_{48}\right)-p_{23}=4 X_{1} X_{4}\left(X_{2} X_{8}+X_{1} X_{4}\right)\left(X_{2}-X_{3}\right)\left(X_{3}-X_{4}\right)\left(X_{4}-X_{2}\right)$, hence the image of the plane is

$$
-(A+B+C+D)^{2} Q R S+B C X_{1} X_{4}\left(X_{2} X_{3}-X_{1} X_{4}\right)\left(X_{2}-X_{3}\right)^{2} S=0
$$

in which $Q, R, S$ are three cubic expressions of the form

$$
X_{3} X_{4}\left(X_{1}+X_{2}\right)-X_{1} X_{2}\left(X_{3}+X_{4}\right), \text { etc. }
$$


The two values of $y_{3}, y_{4}$ are equal if

Hence

$$
\frac{p_{24}^{2}+p_{2 B}^{2}-p_{24}^{2}}{p_{23} p_{24}}= \pm 2
$$

$$
\left[\left(p_{23}+p_{24}\right)^{2}-p_{84}^{2}\right]\left[\left(p_{23}-p_{24}\right)^{2}-p_{34}^{2}\right]=0,
$$

from which we find the surface of branch points to be

$$
L^{\prime}=X_{1} X_{2} X_{3} X_{4}\left(X_{1} X_{4}-X_{2} X_{3}\right)\left(X_{2} X_{4}-X_{3} X_{1}\right)\left(X_{3} X_{4}-X_{1} X_{2}\right)=0
$$

The component surfaces in $L^{\prime}=0$ are the images of the basis points $(1, \pm 1, \pm 1, \pm 1)$, apart from the point $(1,1,1,1)$.

To express $(y)$ in terms of $(X)$ we start from equations similar to (8) but for $y_{2} / y_{1}, y_{3} / y_{1}$, and $y_{1} / y_{1}$. From these we find

$$
\begin{aligned}
y & =Q R S, \\
y_{2}+y_{1} & =\left\{-2 X_{1} X_{2} H_{3} H_{4}+2\left(X_{1}-X_{2}\right) \sqrt{L^{\prime}}\right\} Q \\
y_{3}+y_{1} & =\left\{-2 X_{1} X_{3} H_{2} H_{4}+2\left(X_{1}-X_{3}\right) \sqrt{L^{\prime}}\right\} R, \\
y_{4}+y_{1} & =\left\{-2 X_{1} X_{4} H_{2} H_{3}+2\left(X_{1}-X_{4}\right) \sqrt{L^{\prime}}\right\} S
\end{aligned}
$$

where $H_{2}$ stands for $X_{1} X_{2}-X_{3} X_{4}$, etc.

The equations (8) and (11) express algebraically the $(1,2)$ correspondence between the spaces $(X)$ and $(y)$.

CORNELL UNIVERSITY,

ITHACA, N. Y. 